\title{
Monitoramento do bicho-preguiça Bradypus variegatus Schinz, 1825 (Xenarthra: Bradypodidae) em um remanescente de Floresta Atlântica (João Pessoa-PB, Nordeste do Brasil)
}

\section{Gibran Anderson Oliveira da Silva ${ }^{1 * *}$, Marília Carolina Pereira da Paz $^{1}$, Tarcísio Alves Cordeiro ${ }^{2}$}

${ }^{1}$ Curso de Graduação em Ciências Biológicas. Centro de Ciências Exatas e da Natureza. Universidade Federal da Paraíba. Cidade Universitária. João Pessoa-PB. Brazil. (CEP 58051-900).*E-mail: gibranderson@gmail.com.

${ }^{2}$ Departamento de Sistemática e Ecologia. Centro de Ciências Exatas e da Natureza. Universidade Federal da Paraíba. Cidade Universitária. João Pessoa-PB. Brazil. (CEP 58051-900).

Resumo. A fragmentação dos habitats da Floreta Atlântica ameaça a mastofauna silvestre promovendo isolamentos genéticos das populações, diminuição da variabilidade genética, aumento do risco de doenças, além de afetar a dinâmica dos grupos, comportamentos e área de uso. Encontra-se ameaçada Bradypus variegatus Schinz, 1825 (Xenarthra, Bradypodidae), os bichos-preguiça, presentes nos remanescentes da Universidade Federal da Paraíba (UFPB). Um dos problemas mais comuns com os grupos destes animais está associado aos atropelamentos e morte por queda durante a travessia entre fragmentos, porém ainda são escassos os estudos de manejo e gestão dos animais ocasionando impactos ecológicos proporcionados por manejos equivocados e com a translocação de animais para outras áreas de Floresta Atlântica na Paraíba. A presente pesquisa teve como objetivos verificar e monitorar o uso de espaço por B. variegatus nos fragmentos da UFPB, realizar o levantamento de uso de estratos vegetais e comportamentos exibidos pelos animais nas bordas dos fragmentos com o intuito de promover estratégias eficazes de manejo dos animais. Para isso foram coletados através de um etograma o uso de estratos vegetais e comportamentos exibidos pelos animais nas áreas de bordas dos fragmentos internos da UFPB. A partir destes dados foram obtidos um maior uso para os comportamentos de deslocamento, alimentação, forrageio e agonismo o estrato de subbosque, já os estratos bosques e dosséis foram exclusivos para alimentação, forrageio e deslocamento; enquanto que o solo foi o estrato que mais possuiu comportamentos agonísticos direcionados aos humanos, principalmente a aqueles que manejavam os bichos preguiça. Além disso, foi obtido consumo por uma fêmea adulta de uma espécie de liana (Stigmaphyllon rotundifolium) que recobre parcialmente uma das pontes ecológicas. Outro registro importante foi obtido através de entrevista livre que amostrou fotograficamente uma preguiça fêmea adulta carregando um infante no dorso durante o período noturno, utilizando o sub-bosque de uma árvore isolada no estacionamento do Centro de Ciências Exatas e da Natureza (CCEN). No total, 261 comportamentos e 247 usos de estratos vegetais por

Recebido: $16 / 10 / 2017$

Aceito: $18 / 11 / 2017$

Publicado: 31/12/2017

Corrigido: $31 / 12 / 2018$

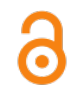

Acesso aberto Artigo completo

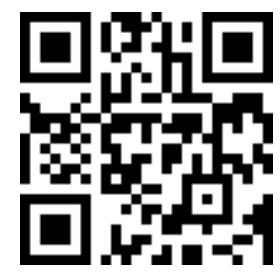

ORCID

(ㄱ) 0000-0001-8451-2123 Gibran Anderson Oliveira da Silva

(D) 0000-0002-3143-7905 Marilia Carolina Pereira da Paz

(1) 0000-0002-9925-3272 Tarcísio Alves Cordeiro 
B. variegatus nas bordas dos fragmentos do Campus I da UFPB. Tais dados associados a novas informações são ferramentas para a elaboração de estratégias de gestão e manejo de animais.

Palavras-chave: Etologia de mamíferos; Pontes ecológicas; Manejo de fauna; Gestão de fauna.

Abstract. Monitoring of Bradypus variegatus Schinz, 1825 (Xenarthra: Bradipodidae) in a remanescent of Atlantic Forest (João Pessoa-PB, Northeast Brazil). A fragmentation of the Atlantic Forest threatens the wild mastofauna, promoting genetic isolation of the populations, diminishing the genetic variability, increasing the risk of diseases, besides affecting the dynamism of the groups, behaviors and area of use. Bradypus variegatus Schinz, 1825 (Xenarthra, Bradypodidae), the sloth who live in the remanescent of the Federal University of Paraiba (UFPB) are threatened. One of the most commom problems with groups of these animals is associated with run-down and death by falling during crossing between fragments, although animal management and management studies are still scarce, causing ecological impacts from mismanagement and animal translocation to other areas of Atlantic Forest in Paraíba. The present research had as objectives to verify and to monitor the use of space by B. variegatus in the fragments of the UFPB; to carry out the survey of the use of vegetal strata and behaviors exhibited by the animals in the borders of the fragments with the intentions to elaborate strategies of conduction of the animals. Therefore it is transmitted through an etogram or use of vegetal strata and behaviors exhibited by the animals in the edge areas of the UFPB internal fragments. Was obtained a greater use for the behaviors of displacement, feeding, foraging and agonism to understory stratum; feeding, foraging and displacement to forest and canopies strata; the ground was the stratum that has agonistic behaviors intended at humans, especially those who handle sloths. And besides, consumption by an adult female of a liana species (Stigmaphyllon rotundifolium) that partially covers one of the ecological bridges was obtained. Another important document was obtained through free interview that photographed a female adult sloth carrying a puppy on the back at night, using the understory of an isolated area of the parking lot in the Center of Exact Sciences and Nature (CCEN). In the total, 261 behaviors and 247 uses of vegetal strata by $B$. variegatus in the edges of the fragments of Campus I of the UFPB. Such data associated to new information are tools for the elaboration of strategies for the management and management of animals.

Keywords: Ethology of mammals; Ecological bridges; Management of fauna; Wildlife management.

\section{Introdução}

A Floresta Atlântica tem sido explorada e fragmentada de modo intenso durante o século $\mathrm{XX}$, principalmente pela intervenção antrópica, como a urbanização
(Saatchi, 2000; Chiarello, 2003; Lagos; Muller, 2007; Rocha et al., 2012). Esta redução e fragmentação de possíveis habitats ameaçam a sobrevivência das populações de animais silvestres. Principalmente através de isolamentos 
genéticos e consequentemente a diminuição da variabilidade genética, que podem causar o acúmulo de homozigotos deletérios de componentes de aptidão, como sobrevivência e fecundidade (Futuyma, 2005; Silva e ValençaMontenegro, 2016).

Além de aumentar o risco de doenças hereditárias (Primack, 2001; Silva; Valença-Montenegro, 2016), as populações isoladas ainda estão sujeitas aos eventos estocásticos, como a variação na razão sexual; baixo sucesso reprodutivo; aleatoriedades ambientais; e alterações comportamentais dos grupos, como na dinâmica, na área de uso e dieta (Primack, 2001; Chiarello, 2003; Castro-Vásquez et al., 2010; Silva e Valença-Montenegro, 2016)

A Universidade Federal da Paraíba (UFPB) em João Pessoa, Campus I, possui uma área localizada entre vários fragmentos de Floresta Atlântica, como a Mata do Buraquinho (329,39 ha) e Bacia do Rio Timbó, que anteriormente formavam uma área continua de floresta. Entretanto estes remanescentes além de fragmentados estão sendo gradativamente perturbados e consequentemente afetando a diversidade local (Medeiros, 2010). Estas áreas representam uma alta importância ao patrimônio biológico nacional devido seu papel ecológico, paisagístico e principalmente pela sua biodiversidade (Santos, 2014; Martin, 2015).

Apenas a área do Campus I da UFPB possui uma riqueza de 456 espécies, sendo 89 destas espécies inclusas na lista vermelha de risco de extinção da União Internacional para Conservação da Natureza (IUCN), contudo a maioria das espécies não apresentam risco de extinção, devido principalmente a capacidade de viverem em ambientes antropizados, como timbus (Didelphis albiventris) e saguis (Callithrix jacchus) (Santos, 2014), no entanto esta aptidão não é garantia da sobrevivência destes animais a longo e curto prazo (Martin, 2015).

Bradypus variegatus Schinz, 1825, também conhecida como bicho-preguiça, está entre as espécies afetadas por pertubações antrópicas, principalmente por atropelamentos (Glista et al., 2009). Encontra-se na Lista Vermelha da IUCN na categoria pouco preocupante (LC) (Medeiros, 2010; Santos, 2014; Martin, 2015). A espécie habita uma ampla distribuição ocorrendo parcialmente desde o sul Honduras passando pelos Andes no Equador, na Venezuela, e na Colômbia seguindo até a Bolívia tendo limites territoriais no norte da Argentina e no Brasil.

Pode-se também encontrar $B$. variegatus geralmente em altitudes de até $1.100 \mathrm{~m}$, utilizando os estratos arbóreos mais elevados e variados na Floresta Atlântica (Pinheiro, 2008; Castro-Vásquez et al., 2010). A distribuição da espécie tem sido reduzida junto com seu ambiente natural devido a alta susceptibilidade da espécie as alterações do habitat, como uma mobilidade limitada, um reduzida área de vida e uma natureza arredia (CastroVásquez et al., 2010; Martin, 2015).

A população de $B$. variegatus do Campus I da UFPB possui uma abundância de 214 indivíduos, uma densidade populacional aproximadamente de 2,26 animais/ha, baixa quando comparados com outros estudos com a espécie em ambientes naturais e urbanos (12,5 animais/ha). A distribuição das preguiças não é homogênea entre os remanescentes e os maiores remanescentes não possuem correlação com uma alta abundância de animais. As espécies vegetais mais consumidas pelos animais visualizados durante uma pesquisa no Campus foram: Cecropia sp. (embaúba), Eriotheca gracilipes (paina-do-campo), Bowdichia virgilioides (sucupira), Tapirira guianensis (cupiúva), Ilex sp., Apuleia leiocarpa (garapeira), Licania octandra (oiticica) e Pterocarpus violaceus (pau sangue) (Martin, 2015).

A utilização de pontes ecológicas para comunicar estes remanescentes florestais pode ser uma das estratégias viáveis para mitigar os efeitos causados pela fragmentação à fauna local (Medeiros, 2010). Possivelmente contribuindo para uma dinâmica de metapopulação que permite recolonizações por migração, reutilização dos habitats, dispersão de espécies vegetais endêmicas, restabeleci- 
mento de ecossistemas, aumento da área de uso e da disponibilidade de recursos acessíveis, como também uma provável redução do isolamento genético (Medeiros, 2010; Silva, 2013; Smith et al., 2015). Apesar das pontes ecológicas proporcionarem tais efeitos de mitigação de danos, ainda existem poucos estudos que promovam o monitoramento e testem a efetividade dessas pontes ecológicas (Corlatti, 2009).

Medeiros (2010) implementou no Campus I da UFPB, junto com a Comissão de Gestão Ambiental (CGA/UFPB) sete pontes de conexão ecológicas que permitem a passagem de pequenos mamíferos como C. jacchus e D. albiventris, e possivelmente B. variegatus (Martin, 2015). Entretanto poucos trabalhos de monitoramento do uso dessas conexões ecológicas por B. variegatus foram realizados. Martin (2015) realizou o estudo do comportamento da espécie nos Campus I da UFPB e registrou uma preguiça se aproximar da ponte ecológica de corda, porém o animal não utilizou a ponte para atravessar $\mathrm{o}$ fragmento.

Ainda segundo Martin (2015), as prováveis explicações pelo fato são a grande distância entre os fragmentos conectados (15 m); o alto custo energético; o tipo de estrutura flexível das pontes ecológicas, que não são viáveis para a morfologia das preguiças; a falta de acuidade visual dos animais (Gilmore et al., 2000). Martin (2015) ainda realizou uma comunicação com 27 parques zoológicos do mundo, obtendo 19 respostas sobre as estruturas das pontes ecológicas. Estes parques zoológicos afirmaram que a espécie apresenta uma preferência por estruturas rígidas e firmes, como troncos de árvores posicionados paralelamente ao chão. Sugerindo pontes ecológicas confeccionadas com troncos de árvores, e para uma longa duração tubos de alumínio, como também um esfregaço de pelos de preguiças nos pontos de acesso, devido 0 comportamento de farejamento dos locais anterior ao acesso e locomoção pelas estruturas.

O monitoramento do uso de borda e padrão de atividades, como estratos vegetais e comportamentos, exibidos pelas preguiças são relevantes para agregar informações sobre as estruturas que possibilitem a elaboração de estratégias viáveis para proporcionar uma efetividade do fluxo dos animais.

De modo que estas estratégias permitam o aumento da dispersão e comunicação dos indivíduos entre os fragmentos, possivelmente promovendo a atenuação dos efeitos do isolamento dos remanescentes, diminuição do número de mortes, a redução no número de atropelamentos da vida silvestre e ações errôneas de manejo de $B$. variegatus na Floresta Atlântica do Campus I da Universidade Federal da Paraíba. Para isso o presente estudo verificou estatisticamente $(\mathrm{P} \leq 5)$ os estratos vegetais utilizados e comportamentos exibidos pelos animais.

Este artigo tem como objetivo verificar e inferir o uso de bordas e o padrão de atividades realizados por Bradypus variegatus nos fragmentos da UFPB (Campus I), para que sejam elaboradas estratégias de gestão e manejo dos animais.

\section{Material e métodos}

\section{Local de estudo}

O estudo foi realizado no Campus I da Universidade Federal da Paraíba (Figura 1), em João Pessoa, que possui cerca de 119 ha (Coordenadas de referência -7.135789, 34.845616), e apresenta área com 13 fragmentos de Floresta Atlântica, estimados em 84 ha, divididos em 44 ha para os fragmentos internos e 39 ha para os remanescentes externos (Rosa e Rosa, 2013). 


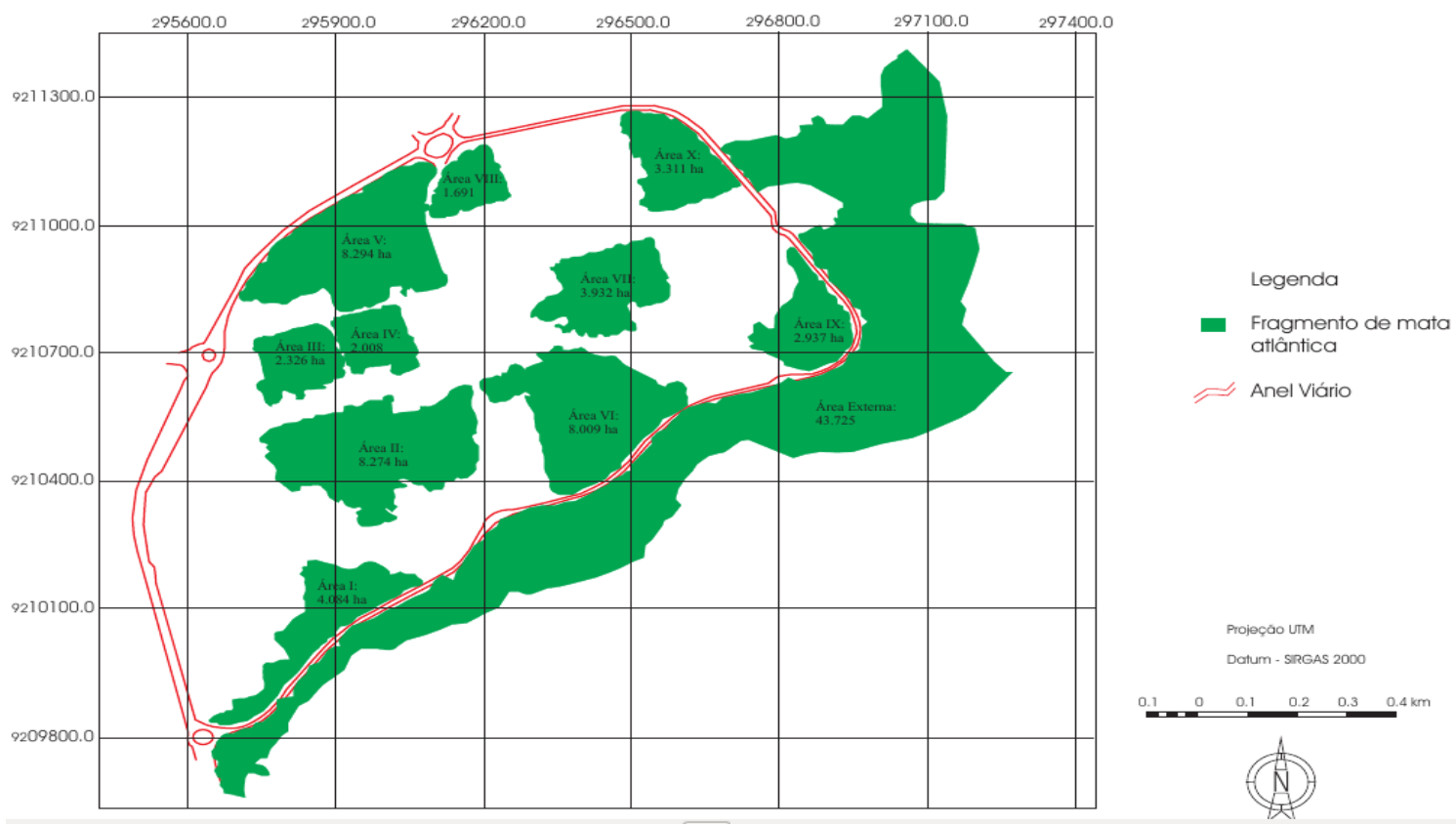

Figura 1. Campus I da Universidade Federal da Paraíba (-7.135789, -34.845616), João Pessoa, Paraíba. Fonte: Rosa e Rosa (2013).

\section{Coletas de dados}

A coleta de dados ocorreu entre os meses de fevereiro e novembro de 2017, cinco dias/mês, em 6 h por dia, totalizando aproximadamente $300 \mathrm{~h}$ de amostragem. Nas quais foram registrados através de etogramas modificados, com base na bibliografia da espécie, os comportamentos exibidos e o uso de estratos vegetais pelos animais, também foi monitorado o uso das pontes ecológicas de cordas pelas preguiças e as demais informações foram obtidas através de entrevistas livres (CastroVásquez et al., 2010; Pedrosa e Castro, 2014; Martin, 2015; Silva e Castro, 2015).

\section{Uso de estratos vegetais}

Para verificar o uso de estrato vegetal foram elaborados etogramas para registrar a altura e as estruturas verticais da vegetação utilizado pelos animais: solo, sub-bosque (1-9 m.), estrato arbóreo (10-15 $\mathrm{m})$ e dossel $(>15 \mathrm{~m})$. A partir destes registros foram calculadas as frequências relativas de uso de cada substrato de acordo com a classe sexo-etária dos indivíduos (Castro-Vásquez et al., 2010; Santos, 2013; Martin, 2015).

\section{Padrão de atividades}

Para o registro dos comportamentos foram utilizados os métodos ad libitum e todas as ocorrências (Altmann, 1974), já para definição das categorias comportamentais foram obtidas através da modificação de etogramas para $B$. variegatus de Castro-Vásquez et al. (2010), Pedrosa e Castro (2014) e Martin (2015).

As categorias de comportamentos elaboradas foram classificadas como: alimentação (A), quando o animal está ingerindo parte de uma planta ou líquen; forrageio $(\mathrm{F})$, quando o animal demonstra uma movimentação com a cabeça e demais membros em busca de determinado alimento e posteriormente o consume; descanso (DC), quando o animal permanece praticamente imóvel coçando o próprio corpo e bocejando; deslocamento (D), quando $o$ animal se movimenta verticalmente e horizontalmente através do estrato utilizando seus membros inferiores e superiores; agonismo (AG), quando o animal arqueia os braços mostrando as garras e possivelmente emitindo vocalização direcionada para predadores ou humanos; social (S), quando o animal exibe interação afiliativa com outros da mesma 
espécie; defecar e/ou urinar (DEF), quando o animal geralmente posicionado imóvel inicia a defecação ou urina; e outros $(\mathrm{O})$, são comportamentos que se diferem das demais categorias elaboradas (Zerda, 2004; Castro-Vásquez et al., 2010; Martin, 2015; Pedrosa e Castro, 2014).

\section{etárias}

Identificação das classes-sexo

Utilizou-se o tamanho, estruturas corporais e sexuais, e o peso dos indivíduos como critérios de definição das classes sexo-etárias macho-adulto, fêmea-adulta, jovens e infantes. Para discernimento do sexo dos indivíduos foi utilizada a presença do espéculo, uma estrutura presente no dorso composto de pelos curtos, negros com as bordas alaranjadas que ocorre exclusivamente machos adultos da espécie (Pinheiro, 2008; Castro-Vásquez et al., 2010; Silva et al., 2013).

Para as demais classes sexo-etárias, como os infantes, foram considerados aqueles animais encontrados permanente ou semipermanentemente associados ao dorso ou ventre de outro indivíduo, pesando $<1,2$ kg; os jovens, são indivíduos visualizados sem associação a outros animais, possuem um comprimento corporal $<40$ centímetros pesando aproximadamente 1,2-2,3 kg; e indivíduos adultos, apresentam entre 40-75 centímetros de comprimento de corporal pesando a partir 2,3 kg (Zerda, 2004; Castro-Vásquez et al., 2010).

\section{Análise dos dados}

Os dados coletados analisados através do teste qui-quadrado com correção em Yates no software R ( $\mathrm{R}$ CoreTeam, 2017), para verificar hipóteses da existência de uma diferença significativa, ou seja diferente do acaso, do uso de estratos de vegetação e comportamentos exibidos de acordo com as classes sexo-etárias, machos-adultos, fêmeas-adultas, jovens e infantes.

Também foram comparados com o comportamento e uso de estratos vegetais de populações de $B$. variegatus em outros estudos na UFPB (Martin, 2015), em ambientes urbanos (Pedrosa e Castro, 2014), semi-urbanos (Urbani e Bosque,
2006; Silva et al., 2013) e ambientes naturais (Castro-Vasquez et al., 2010; Pedrosa e Castro, 2014).

\section{Resultados e discussão}

Durante a pesquisa foram registradas 101 preguiças, destas destacamse 68 fêmeas e 43 machos, totalizando 2,29 ind/ha em todo remanescente interno. Diferente e abaixo do número de indivíduos obtidos no estudo de Martin (2015), que amostrou uma abundância populacional de 214 indivíduos, no Campus I da UFPB. Porém, deve-se considerar que a presente pesquisa foi realizada em curto prazo e foram percorridas apenas as áreas de borda dos remanescentes. Portanto, possivelmente está é a razão da densidade populacional obtida ter sido baixa para a espécie em ambientes naturais e urbanos (12,5 ind/ha) e parcialmente similar à densidade do fragmento interno e externo da UFPB (2,26 ind/ha) (Martin, 2015).

Martin (2015) ainda reforça que a distribuição dos indivíduos em grandes remanescentes não correspondem à uma correlação significativa de uma maior abundância de $B$. variegatus e vice-versa. Outro dado importante obtido foi a morte de três preguiças em junho de 2017. Tal acontecimento reforça a possível hipótese de uma diminuição do número populacional de indivíduos na área de estudo, podendo estar associada às doenças, à falta de conectividade e a morte por senescência dos animais. Estas informações foram obtidas através de entrevistas livres e os indivíduos foram coletados e direcionados por transeuntes ao Laboratório de Mastozoologia do CCEN/UFPB.

Foram registrados 261 comportamentos e 247 usos de estratos vegetais por $B$. variegatus nas bordas dos fragmentos do Campus I da UFPB. O deslocamento (46,71\%), descanso (20,31\%) e alimentação $(19,92 \%)$ foram os comportamentos que mais foram amostrados (Figura 2). Enquanto à alimentação (21\%), o forrageio (11\%) e o agonismo (6\%) foram mais amostrados entre fêmeas da população. $O$ descanso (21\%) e o deslocamento (52\%) foram mais obtidos entre os machos (Figura 3). 


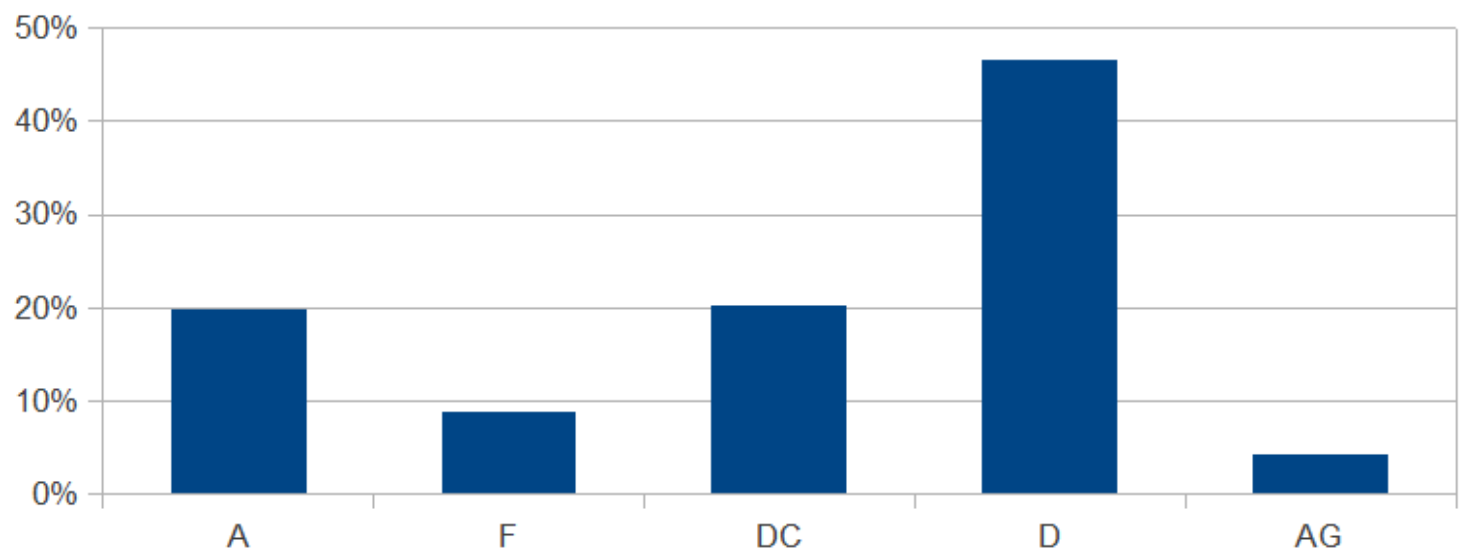

Figura 2. Frequência relativa de comportamentos exibidos por Bradypus variegatus nas bordas do Campus I da UFPB. (A = Alimentação; F = Forrageio; $\mathrm{DC}=$ Descanso; $\mathrm{D}=$ Deslocamento; $\mathrm{AG}=$ Agonismo).

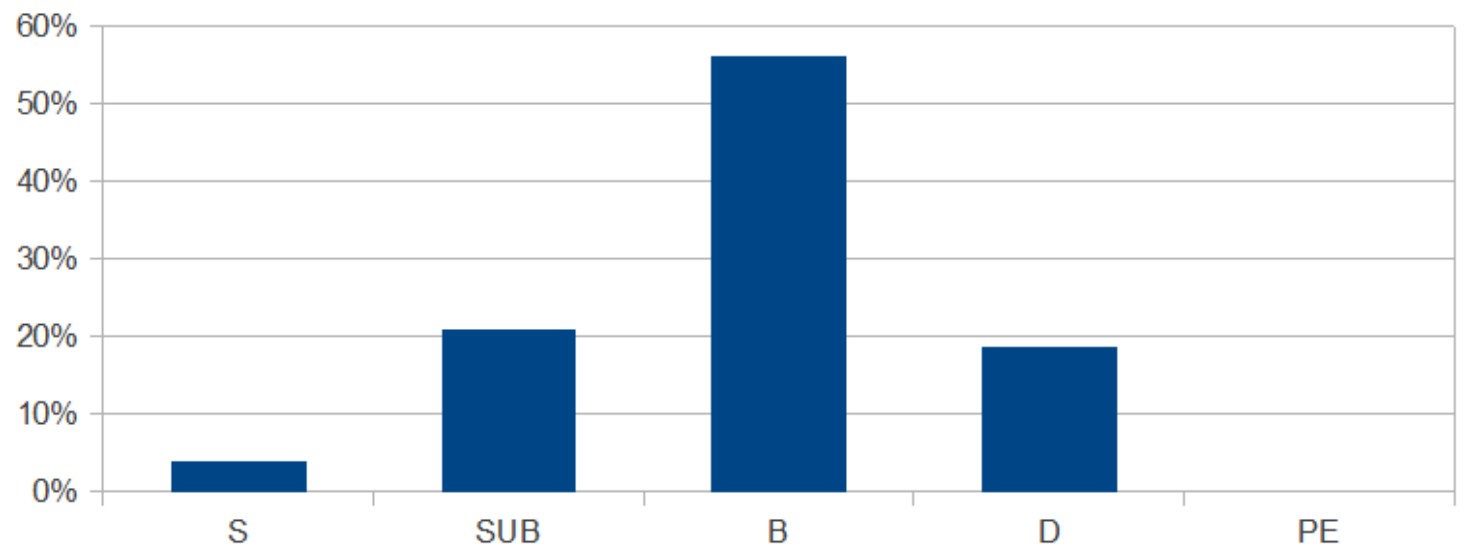

Figura 3. Frequência relativa do uso de estratos vegetais exibidos por $B$. variegatus nas bordas do Campus I da UFPB. (S = Solo; Sub = Sub-bosque; B = Bosque; D = Dossel; PE = Pontes ecológicas).

A alimentação e o forrageio, exibidos majoritariamente pelas fêmeas, podem estar relacionados com a variação na ingestão de nutrientes associada ao sexo, à idade, e à produção hormonal, como também aos períodos reprodutivos (Pinheiro, 2008). Este último fator pode justificar o maior registro de agonismo entre as fêmeas direcionado aos seres humanos. Além disto, o sexo feminino foi o mais amostrado durante todo o estudo utilizando, principalmente, o solo e carregando filhotes (Figura 4). Os agonismos obtidos foram direcionados exclusivamente aos espectadores e transeuntes que manejavam os animais, geralmente de modo errôneo. Enquanto que o deslocamento, realizado majoritariamente pelos machos, podem estar associados ao movimento de dispersão em busca de parceiras sexuais ou pressões ambientais dos fragmentos (Figura 5). 


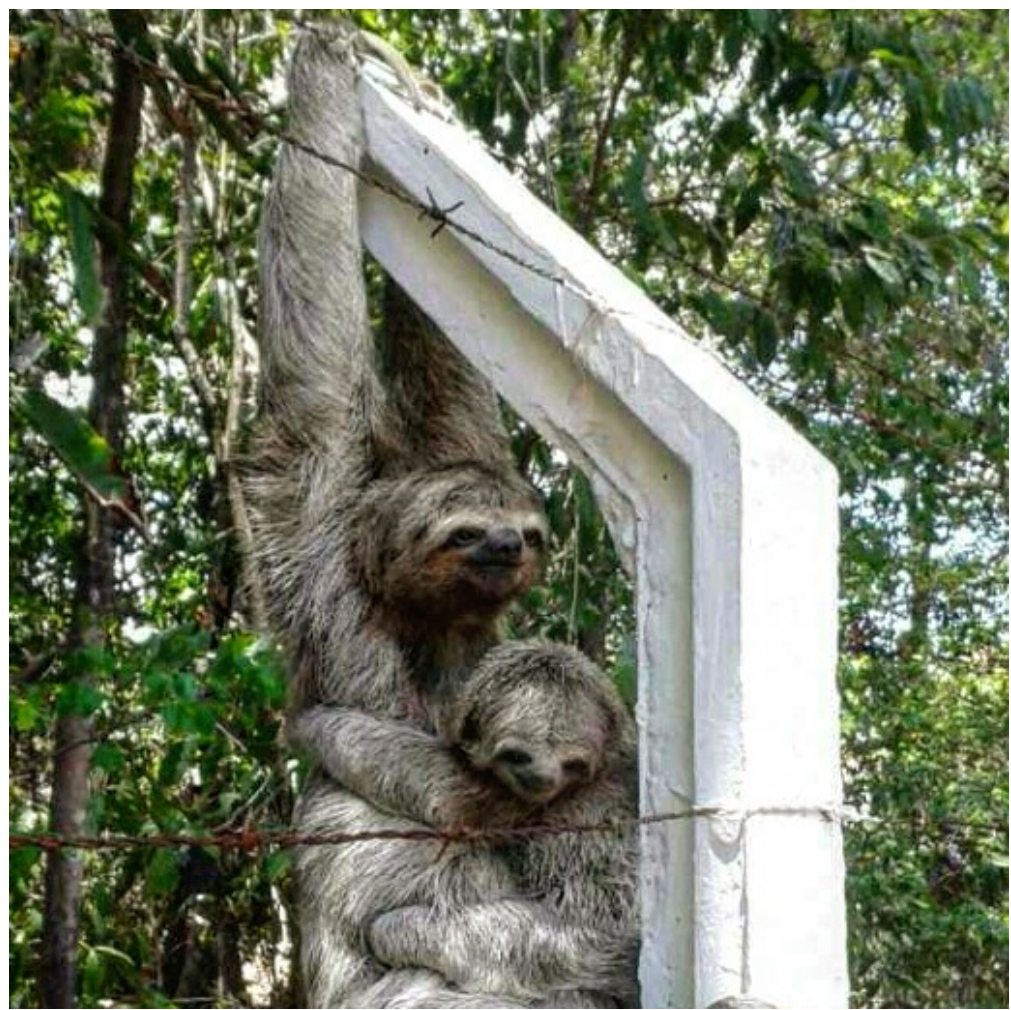

Figura 4. B. variegatus fêmea adulta carregando um filhote do solo para o sub-bosque da área de borda Campus I da UFPB. Fonte: Comissão de Gestão Ambiental da UFPB.

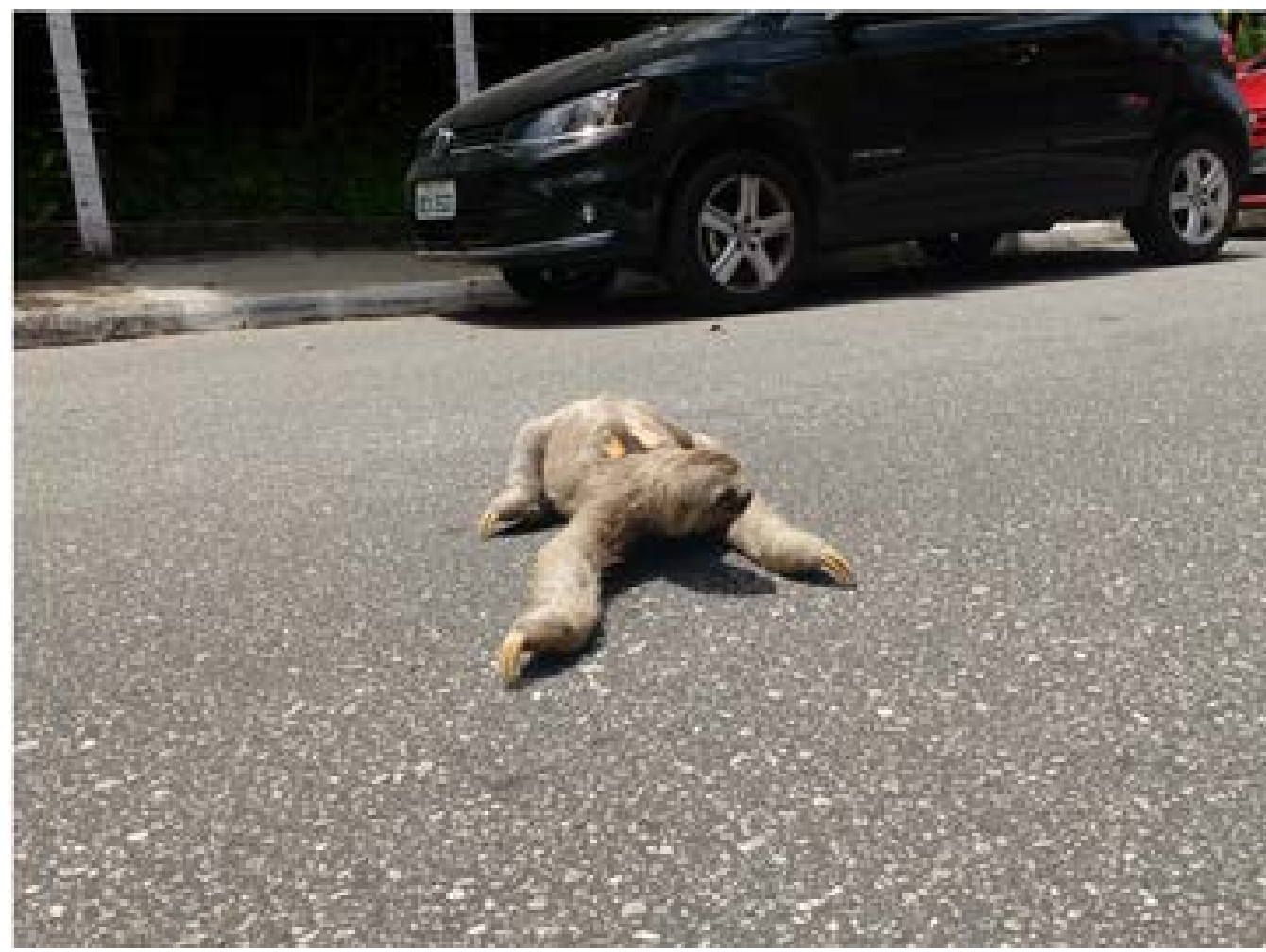

Figura 5. Indivíduo macho adulto B. variegatus se deslocando no solo do Campus I, da UFPB. 
Os estratos vegetais mais destacados foram bosque (56,28\%), dossel $(21,05 \%)$ e sub-bosque $(18,62 \%)$. As pontes ecológicas não foram utilizadas pelos animais durante todo o estudo (Figura
3). Para os estratos de vegetação, o solo $(5,80 \%)$ e o bosque $(57,25 \%)$ foram mais utilizados por fêmeas, enquanto o subbosque $(22,94 \%)$ e o dossel $(20,18 \%)$ foram mais usados pelos machos (Figura 6).

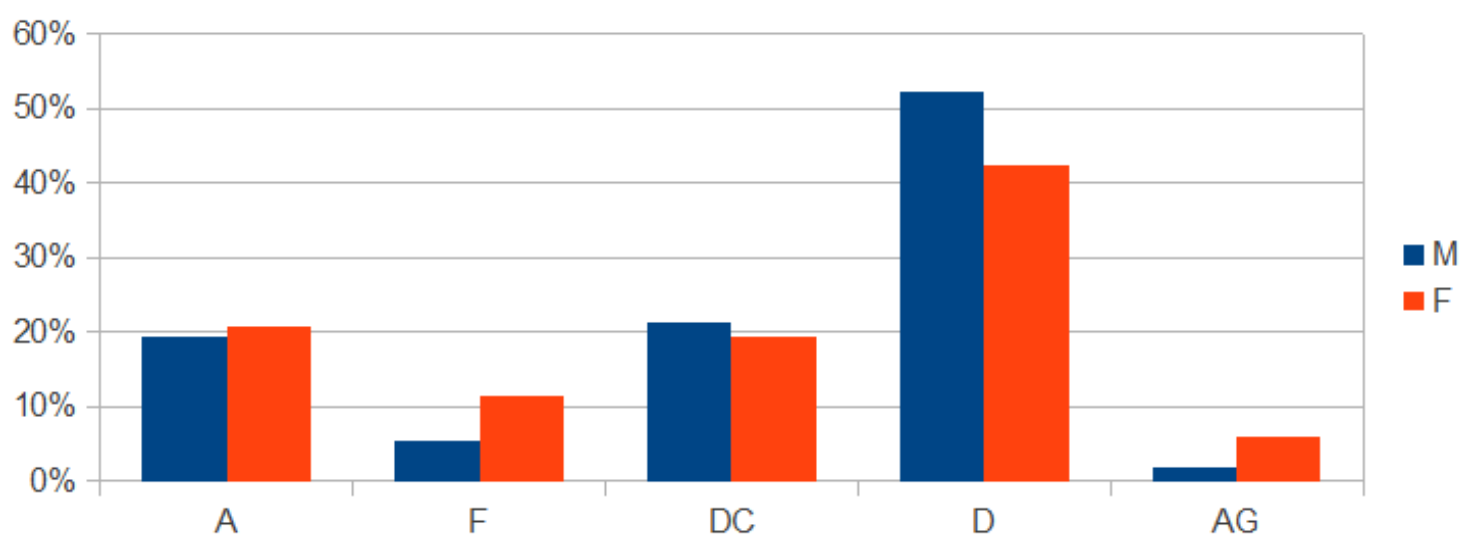

Figura 6. Frequências relativas de comportamentos exibidos pelos indivíduos machos e fêmeas de B. variegatus nas bordas do Campus I da UFPB. (A=Alimentação; F= Forrageio; DC=Descanso; $\mathrm{D}=$ Deslocamento; $\mathrm{AG}=$ Agonismo; F=Fêmea; $\mathrm{M}=$ Macho).

\begin{abstract}
Os comportamentos e os estratos de vegetação destacados podem ser justificados possivelmente devido aos contextos em que os animais foram encontrados. Tais eventos ocorreram, na maioria das vezes, durante o forrageamento e a obtenção de recursos alimentares pelos animais através de espécies vegetais pioneiras, tais como Cecropia sp. (embaúba), Bowdichia virgilioides (sucupira), Tapirira guianensis (cupiúva) e Licania octandra (oiticica). Além destas espécies serem utilizadas como meio de dispersão também já foram utilizadas na dieta da espécie na área de estudo (Martin, 2015).
\end{abstract}

Estudos sobre a eficiência digestiva em preguiças verificaram que indivíduos B. variegatus preferem folhas mais novas e brotos, por possuírem uma maior digestibilidade devido à composição química, por isso os animais aparentemente selecionam árvores que possuem um alto volume de copa, uma alta exposição solar e abundância de cipós (Montgomery e Sunquist, 1978; Pinheiro, 2008).

Tais informações corroboram com os dados obtidos durante a atual pesquisa, onde o forrageio $(21,33 \%)$ e a alimentação (44\%) foram registradas majoritariamente no bosque, como também no sub-bosque, tendo em vista que nas áreas de estudo ocorrem poucas árvores com estrutura e altura dossel (Figura 7). Estes mesmos estratos vegetais também foram essenciais para os deslocamentos, sendo $43 \%$ executados no bosque e $26 \%$ no subbosque. Enquanto os comportamentos agonísticos foram mais frequentes no solo (76,92\%) e sub-bosque $(23,08 \%)$. Logo é possível inferir que os estratos de vegetação bosque e sub-bosque são essenciais para atividades primordiais para os animais, como alimentação, forrageio e deslocamento (Figura 8). 


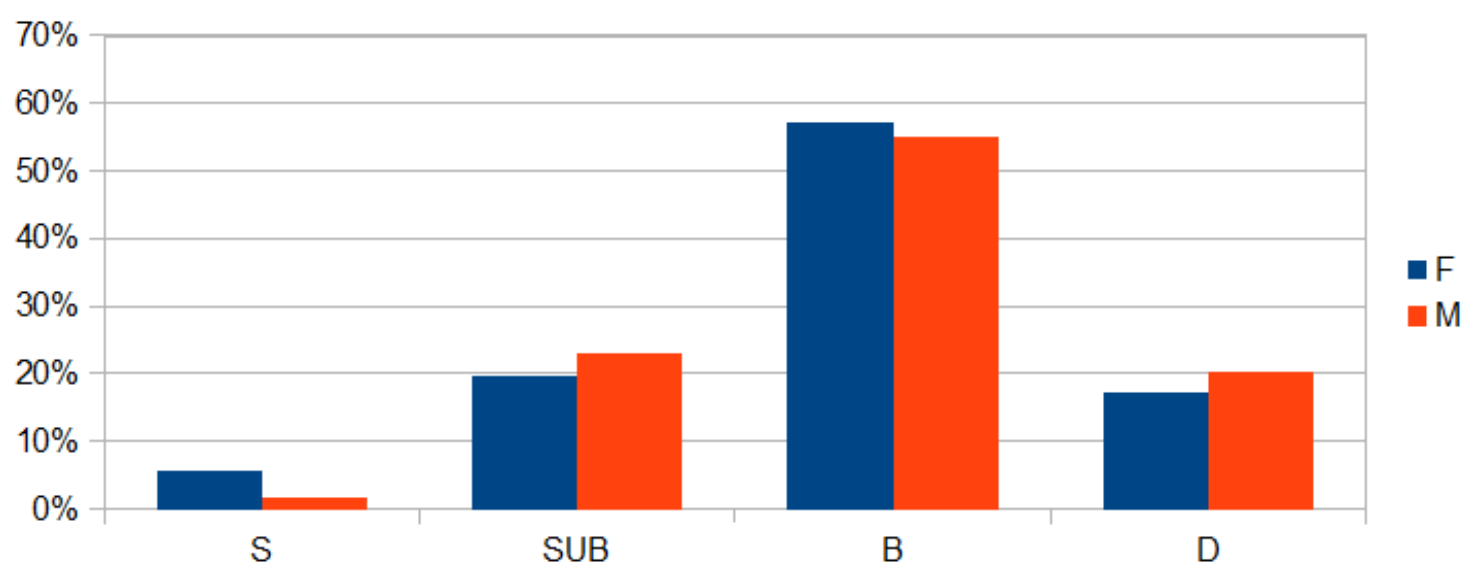

Figura 7. Frequências relativas do uso de estratos vegetais exibidos pelos indivíduos machos e fêmeas de B. variegatus nas bordas do Campus I da UFPB. ( $\mathrm{S}=$ Solo; Sub = Sub-bosque; $\mathrm{B}=$ Bosque; $\mathrm{D}=$ Dossel; $\mathrm{PE}=$ Pontes ecológicas; $\mathrm{F}=$ Fêmea; $\mathrm{M}=$ Macho) .

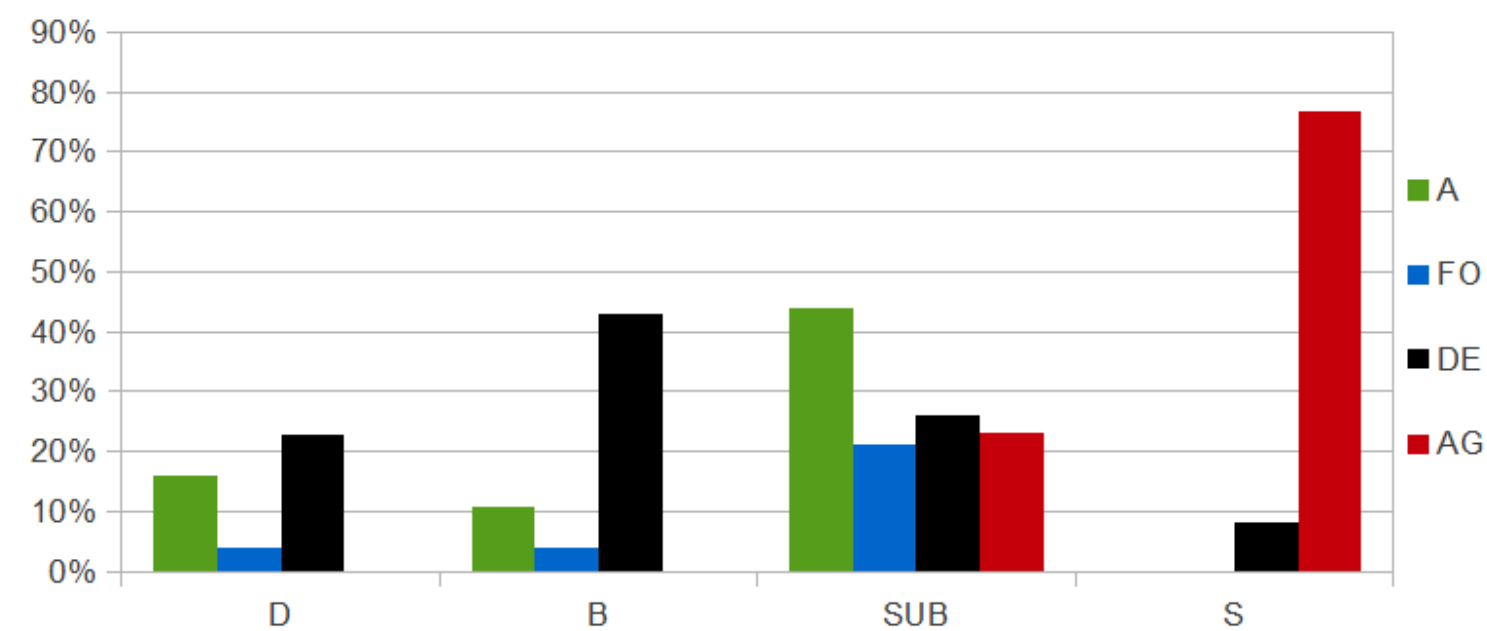

Figura 8. Frequências relativas do uso de estratos vegetais e comportamentos exibidos por B. variegatus no Campus I da UFPB. ( $\mathrm{S}=$ Solo; SUB = Sub-bosque; $\mathrm{B}=$ Bosque; $\mathrm{D}=$ Dossel; A = Alimentação; $\mathrm{FO}=$ Forrageio; $\mathrm{DE}=$ Descanso; $\mathrm{AG}=$ Agonismo $)$.

Apesar do sub-bosque ser o estrato com mais categorias comportamentais exibidas pode-se inferir que é um dos quais as preguiças mais exibem comportamentos agonísticos e consequemente estresse físico. Portanto pode-se levantar a hipótese de que a altura e estruturas dos estratos de subbosque e bosque possuem potencial para instalação viável de pontes ecológicas específicas para $B$. variegatus, junto com um contínuo monitoramento.

Enquanto que o solo é o estrato que mais provoca estresse nos animais devido, principalmente, a intervenção humana durante a presença dos animais nessa estrutura. Pedrosa e Castro (2014) relatam que os bichos-preguiça da mesma espécie em uma área de praça pública no município de Rio Tinto eventualmente não descem ao solo nem até as bases das árvores para defecar e urinar. Possivelmente isso pode ocorrer devido um alto nível de estresse gerado por perturbações humanas durante as atividades dos animais. Além disso a espécie é considerada altamente vulnerável nesse estrato devido a predação por outros 
animais, como corujas (Moreno e Plese, 2006).

Estudo de Lara-Ruiz e SrbekAraujo (2006) e Pinheiro (2008) relatam uma dieta de B. variegatus composta 99\% por folhas e uma preferência por brotos e folhas jovens obtidas tanto de espécies arbóreas quanto de lianas, totalizando 21 espécies no geral. Entretanto numa análise de seleção ao nível individual ocorreram variações, onde o número total de espécies vegetais utilizadas para consumo diminuiu para sete. As espécies mais consumidas foram Micropholis venulosa (Sapotaceae), Ficus sp (Moraceae) e a liana Mandevilla sp (Apocinaceae). Outros estudos também citam áreas com cerca de 91 espécies presentes no Panamá, dessas espécies apenas 30 spp estavam no conteúdo estomacal dos indivíduos e $40 \mathrm{spp}$. foram relatadas sendo consumidas pelos animais (Pinheiro, 2008).

Um registro inédito na área de estudo foi o uso de lianas, da espécie Stigmaphyllon rotundifolium, que recobrem boa parte dos fragmentos do Campus I da UFPB. Além de serem utilizadas para o deslocamento e forrageio dos indivíduos da população, também foram amostradas, sendo consumidas pelos animais nos demais estratos vegetais, como: subbosque, bosque e dossel, exceto o solo (Figura 9).

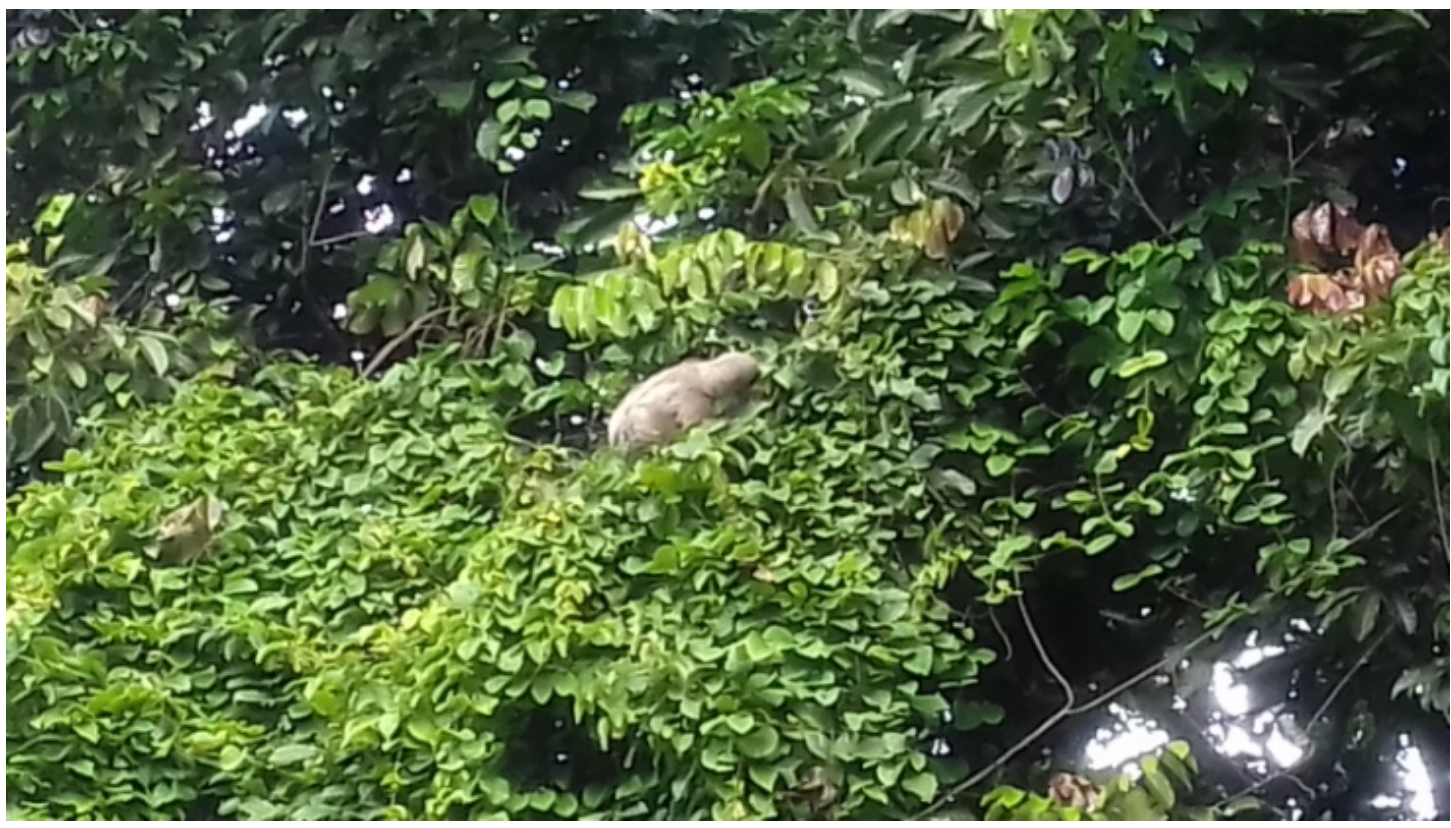

Figura 9. B. variegatus fêmea adulta consumindo Stigmaphyllon rotundifolium no sub-bosque da área de borda Campus I da UFPB.

Provavelmente o consumo de espécies vegetais de $B$. variegatus local é afetado pela disponibilidade e características dos recursos, como o odor, além da ação dos mecanismos fisiológicos em resposta aos alimentos consumidos (Pinheiro, 2008). Dessa forma os fatores evolutivos, sociais, ambientais e estímulos sensoriais podem influenciar o consumo e a preferência alimentar dos indivíduos pelas espécies vegetais registradas. Provavelmente $S$. rotundifolium está relacionada a estes estímulos e pode ser utilizada estrategicamente para a montagem de pontes ecológicas viáveis para $B$. variegatus. Uma das pontes ecológicas de cordas para 
marsupiais e primatas entre fragmentos do Restaurante Universitário da UFPB Campus I apresenta-se recoberta parcialmente por essa espécie.

Outro registro importante obtido através de entrevista livre foi o uso do solo e do sub-bosque de um árvore isolada, por uma preguiça fêmea adulta carregando um filhote no período da noite (29 de novembro de 2016, às 20:45 h) (Figura 10). Na área do estacionamento do CCEN, onde não existem conectividades com os fragmentos maiores.

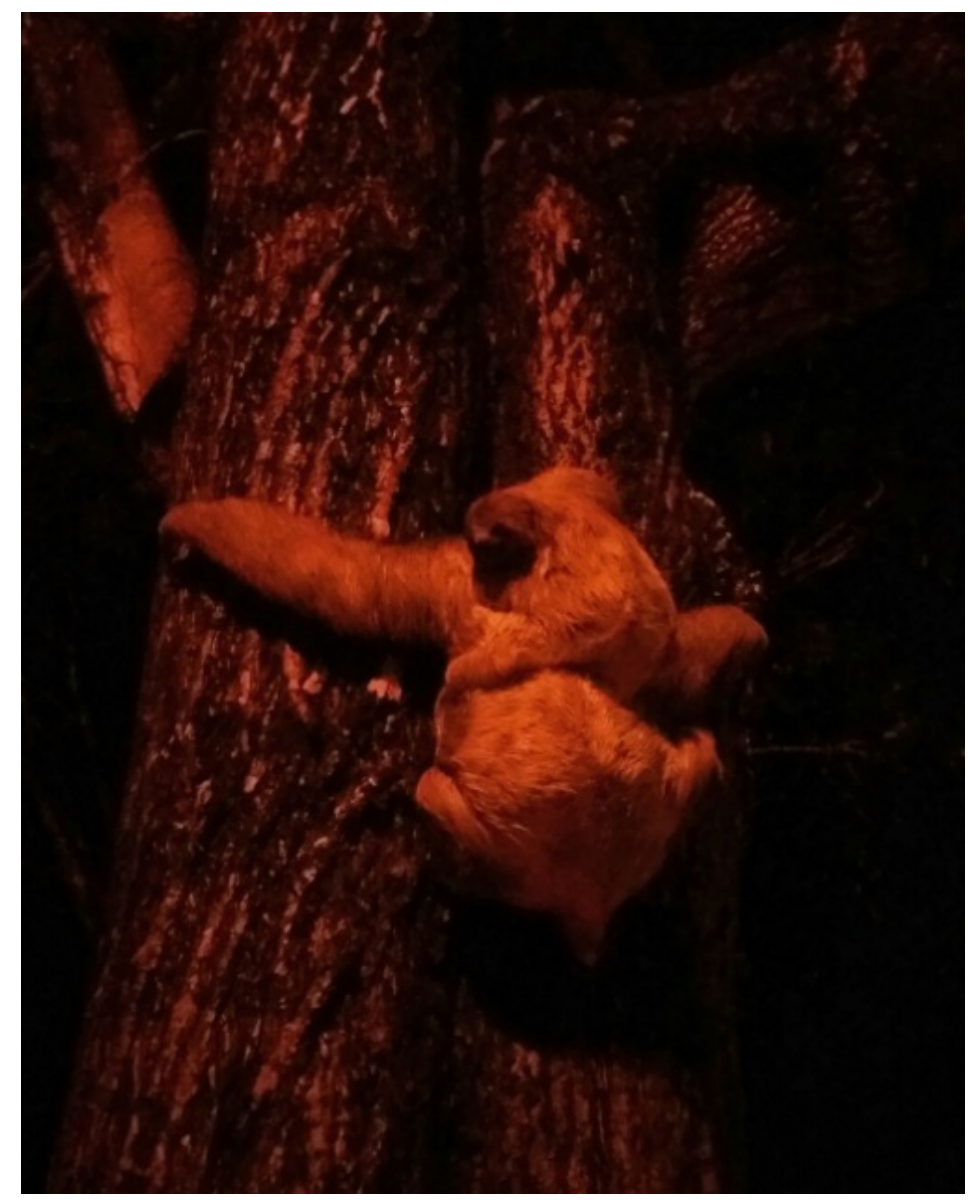

Figura 10. B. variegatus fêmea adulta carregando um filhote do solo para o sub-bosque no período noturno no Campus I da UFPB.

Estudos do padrão de movimentação e de atividade da espécie por Sunquist e Montgomery (1973) e Queiroz (1995) amostraram frequências regulares de atividade para $B$. variegatus, tanto durante o dia como a noite. Resultados similares foram obtidos por Castro-Vásquez (2010) que constatou B. variegatus movimentando-se significativamente durante 0 período noturno e descansando durante o dia, particularmente relacionado com digestão e saciação após alimentação e forrageio. Portanto é importante inferir que um monitoramento para espécie na área deva também proceder durante o período noturno, onde aparentemente esses animais exercem normalmente suas atividades. 


\section{Considerações finais}

Verificou-se que os estratos vegetais bosque, dossel e sub-bosque foram usados com mais frequência pelos animais. $\mathrm{O}$ solo e o bosque foram mais utilizados por fêmeas, e o uso destes está relacionado com os comportamentos de alimentação, forrageio e agonismo, tendo este último forte ligação com o manejo por humanos. Em contrapartida, o sub-bosque e o dossel foram mais usados pelos machos, o que pode estar associado à dispersão reprodutiva ou pressões ambientais que o animal esteja sofrendo nos fragmentos.

Não foi detectado o uso das pontes ecológicas pelos animais, porém foi observado que uma das pontes para marsupiais e primatas encontra-se parcialmente recoberta pela liana Stigmaphyllon rotundifolium, que recobre boa parte da borda dos fragmentos estudados e serve para deslocamento e forrageio, sendo também observado o seu consumo por $B$. variegatus durante 0 estudo.

No total, foram registrados 261 comportamentos e 247 usos de estratos vegetais por $B$. variegatus nas bordas dos fragmentos do Campus I da UFPB, tais dados associados a novos dados obtidos, como o uso para consumo, forrageio e locomoção de $S$. rotundifolium, são ferramentas para a elaboração de estratégias para a gestão e manejo dos animais.

\section{Declaração de conflito de interesses}

Os autores declaram não haver conflito de interesses.

\section{Referências}

Altmann, J. Observational study of bahaviour: sampling methods. Behaviour, v. 49, p. 227267, 1974.

Castro-Vásquez, L.; Meza, M.; Plese, T.; Moreno-Mora, S. Activity patterns, preference and use of floristic resources by Bradypus variegatus in a tropical dry forest fragment, Santa Catalina, Bolívar, Colombia. Edentata,

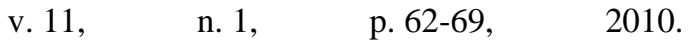
https://doi.org/10.1896/020.011.0111
Chiarello, A. G. Primates of the Brazilian Atlantic Forest: the influence of forest fragmentation on survival. In: Marsh, L. K. (Ed.). Primates in fragments. New York: Kluwer Academic/Plenum Publishers, 2003. p. 99-122.

Corlatti, L.; Hackländer, K.; Frey-Roos, F. Ability of wildlife overpasses to provide connectivity and prevent genetic isolation. Conservation Biology, v. 23, n. 3, p. 548-556, $2009 . \quad$ https://doi.org/10.1111/j.15231739.2008.01162.x

Futuyma, D. J. Evolution. Sunderland: Sinauer Associates, 2005. v. 4. (SeaView version).

Gilmore, D. P.; Da-Costa, C. P.; Duarte, D. P. F. An update on the physiology of two-and three-toed sloths. Brazilian Journal of Medical and Biological Research, v. 33, n. 2, p. 129146, $2000 . \quad$ https://doi.org/10.1590/S0100879X2000000200001

Glista, D. J.; Devault, T. L.; Dewoody, J. A. A review of mitigation measures for reducing wildlife mortality on roadways. Landscape and Urban Planning, v. 91, n. 1, p. 1-7, 2009. https://doi.org/10.1016/j.landurbplan.2008.11.0 01

Lagos, A. R.; Muller B. L. A. Hotspot brasileiro Mata Atlântica. Saúde \& Ambiente em Revista, v. 2, n. 2, p. 35-45, 2007.

Lara-Ruiz, P.; Srbek-Araujo, A. C. Comportamento potencialmente reprodutivo da preguiça-comum, Bradypus variegatus (Xenarthra, Bradypodidae): observações de campo. Edentata, n. 7, p. 44-46, 2006. https://doi.org/10.1896/1413-4411.7.1.44

Martin, A. Behavior of Bradypus variegatus in an urban area (UFPB Campus, João Pessoa, Brazil). Paris: Université Paris 13 - UFR Lettres, Sciences de L'Homme et des Sociétés, 2015.

Medeiros, S. T. A. Proposta para implantação de estruturas para a conexão ecológica entre os fragmentos florestais do Campus I da UFPB e do seu entorno. João Pessoa: Universidade Federal da Paraíba, 2010. (Monografia).

Moreno, S.; Plese, T. The illegal traffic in sloths and threats to their survival in Colombia. $\begin{array}{llll}\text { Edentata, } & \text { n. 7, } & \text { p. 10-18, } 2006 .\end{array}$ https://doi.org/10.1896/1413-4411.7.1.10

Nagy, K. A.; Montgomery, G. G. Field metabolic rate, water flux, and food consumption in three-toed sloths (Bradypus variegatus). Journal of Mammalogy, v. 61, 
n. 3

p. $465-472$,

1980.

https://doi.org/10.2307/1379840

Pedrosa, E. P.; Castro, C. S. S. Behavior patterns of the common sloth (Bradypus variegatus Schin, 1825) in urban and natural environments in Rio Tinto, Paraíba, State, Brazil. Brazilian Journal of Ecology, v. 16, n. 1/2, p. 114-122, 2014. Disponível em: <http://seb-ecologia.org.br/revistas/2014/12.pdf > . Acesso em: 23 ago. 2016.

Pinheiro, S. D. Comportamento alimentar da preguiça comum Bradypus variegatus Schinz, 1825 (Xenarthra, Bradipodidae) no Parque Centenário de Barra Mansa-RJ. Juiz de Fora: Universidade Federal de Juiz de Fora, 2008. (Dissertação).

Primack, R. B.; Rodrigues, E. Biologia da Conservação. Londrina: Vida, 2001.

Queiroz, H. L. Preguiças e guaribas, os mamíferos folívoros arborícolas do Mamirauá. Brasília: CNPq/Sociedade Civil Mamirauá, 1995.

R Core Team. R: A language and environment for statistical computing. Vienna, Áustria: R Foundation for Statistical Computing, 2017. Disponível em: <https://www.R-project.org/>. Acesso em: 23 ago. 2016.

Rocha, E. C.; Silva, E.; Dalponte, J. C.; Del Giúdice, G. M. L. Efeito das atividades de ecoturismo sobre a riqueza e a abundância de espécies de mamíferos de médio e grande porte na região do Cristalino, Mato Grosso, Brasil. Revista Árvore, v. 36, n. 6, p. 1061-1072, $2012 . \quad$ https://doi.org/10.1590/S010067622012000600007

Saatchi, S.; Agosti, D.; Alger, K.; Delabie J.; Musinsky J. Examining fragmentation and loss of primary forest in the Southern Bahian Atlantic Forest of Brazil with radar imagery. Conservation Biology, v. 15, n. 4, p. 867-875, 2001. 1739.2001.015004867.x https://doi.org/10.1046/j.1523-

Santos, A. C. A. Padrão de forrageamento de Cebus flavius (Schreber, 1774) (Primates: Cebidae), em um fragmento de Mata Atlântica na Paraíba. São Cristóvão: Universidade Federal de Sergipe, 2013. (Dissertação).

Santos, T. O. Riqueza e status de conservação de espécies no Campus I da UFPB, João
Pessoa, Paraíba, Brasil. João Pessoa: Universidade Federal da Paraíba, 2014. (Monografia).

Silva, C. P.; Castro, C. S. S. Percepção dos frequentadores da Praça João Pessoa em Rio Tinto, PB, quanto aos impactos do ambiente urbano no bicho preguiça (Bradypus variegatus) e ações de educação ambiental. Educação Ambiental em Ação, v. 14, n. 54, 2015. Disponível em: <http://www.revistaea.org/ pf.php?idartigo=2232>. Acesso em: 23 ago. 2016.

Silva, G. A. O.; Valença-Montenegro, M. M. Ecologia alimentar e nutricional de Sapajus flavius como critério de escolha de áreas para reintrodução da espécie, contribuição: Estação Experimental de Camaratuba. Anais do VIII Seminário de Pesquisa e Iniciação Científica do Instituto Chico Mendes de Conservação da Biodiversidade, Brasília, 2016. 1 CD-ROOM.

Silva, S. M.; Clozato, C. L.; Moraes-Barros, N.; Morgante, J. S. Towards a standard framework to describe behaviors in the common-sloth (Bradypus variegatus Schinz, 1825): novel interactions data observed in distinct fragments of the Atlantic Forest, Brazil. Brazilian Journal of Biology, v. 73, n. 3, p. 527-531, $2013 . \quad$ https://doi.org/10.1590/S151969842013000300010

Silva, V. D. Diversidade e composição de árvores usadas como recurso por primatas em fragmentos de Mata Atlântica. Rio Tinto: Universidade Federal da Paraíba, 2013. (Monografia).

Smith, D. J.; Van Der Ree, R.; Rosell, C. Wildlife crossing structures. In: Van Der Ree, R.; Smith, D. J.; Grilo, C. Handbook of Road Ecology: an effective strategy to restore or maintain wildlife connectivity across roads. New York: Wiley, 2015. p. 172-183. https://doi.org/10.1002/9781118568170.ch21

Sunquist, M. E.; Montgomery, G. G. Activity patterns and rates of movement of two-toed and three-toed sloths (Choloepus hoffmanni and Bradypus infuscatus). Journal of Mammalogy, $\begin{array}{llll}\text { v. } 54, & \text { n. 4, } & \text { p. 946-954, }\end{array}$ https://doi.org/10.2307/1379088

Zerda, E. Comportamiento animal: introducción, métodos y prácticas. Bogotá: Editorial Unibiblos, 2004. 